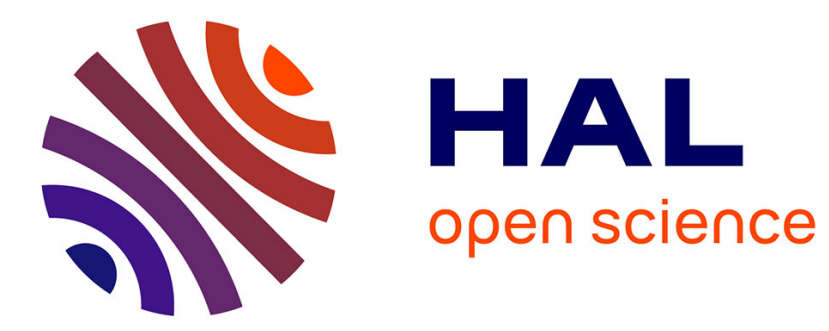

\title{
Immersive moodboards, a comparative study of industrial design inspiration material
}

\author{
Vincent Rieuf, Carole Bouchard, Améziane Aoussat
}

\section{To cite this version:}

Vincent Rieuf, Carole Bouchard, Améziane Aoussat. Immersive moodboards, a comparative study of industrial design inspiration material. 2017. hal-01096968

\section{HAL Id: hal-01096968 \\ https://hal.science/hal-01096968}

Preprint submitted on 14 Feb 2017

HAL is a multi-disciplinary open access archive for the deposit and dissemination of scientific research documents, whether they are published or not. The documents may come from teaching and research institutions in France or abroad, or from public or private research centers.
L'archive ouverte pluridisciplinaire HAL, est destinée au dépôt et à la diffusion de documents scientifiques de niveau recherche, publiés ou non, émanant des établissements d'enseignement et de recherche français ou étrangers, des laboratoires publics ou privés. 


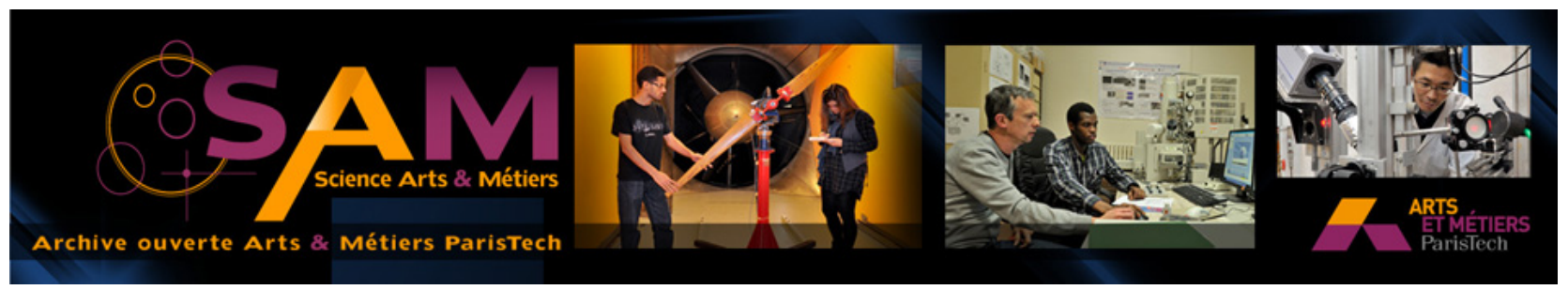

Science Arts \& Métiers (SAM)

is an open access repository that collects the work of Arts et Métiers ParisTech researchers and makes it freely available over the web where possible.

This is an author-deposited version published in: http://sam.ensam.eu

Handle ID: .http://hdl.handle.net/10985/9117

\section{To cite this version :}

Vincent RIEUF, Carole BOUCHARD, Améziane AOUSSAT - Immersive moodboards, a comparative study of industrial design inspiration material - Journal of Design Research - Vol. Fothcoming, nFothcoming, p.Fothcoming - 2016 


\title{
Immersive moodboards, a comparative study of industrial design inspiration material
}

\author{
Vincent Rieuf, Carole Bouchard, Améziane Aoussat
}

Laboratoire Conception de Produits et Innovation, Arts et Métiers ParisTech, 151, Boulevard de l'hôpital - 75013 Paris, France

A recent trend in our industrial culture has been the gradual emergence of digital tools in various fields of human activity. They aim to reduce development time, cost, or to insure a low error, high quality process. Many fields have been improved thanks to this new computerized approach. This paper is centered on how industrial design could be assisted by virtual reality tools.

More specifically it presents a new vision of early design methodologies through immersive technologies. It also presents the results of an experimentation aiming to compare traditional moodboards with a newly developed immersive moodboard.

When analyzing and comparing the relationship of the industrial designer to a traditional and an immersive moodboard, our result highlights the fact that immerging the industrial designer in an immersive moodboard induces a high emotional activity without radically modifying the meaning of the represented trend. The moodboard data spatialization stimulates and engages the designer into interacting with the immersive moodboard. The virtual reality system provides the illusion of a potential reality, which can be used by the designer as a reflection basis for his work. We believe that delivering this immersive experience during the early design process will help the industrial designer make style related decisions.

Keywords - Cognitive Psychology, Experience, Immersion, Industrial Design, Inspirational Trends, Virtual Reality

Relevance to Design Practice - We propose and have evaluated immersive moodboards dedicated to the assessment of a trend during the early product design. We have found that merging the designers' reality with an imaginary spatialized trend induces a higher emotional impact. We aim to help early decisions regarding the products' style. 


\section{Sommaire}

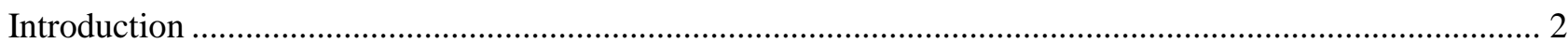

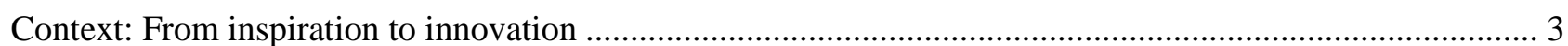

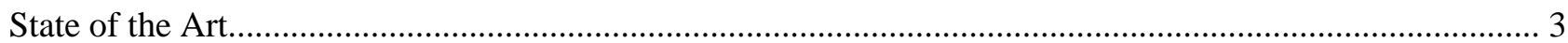

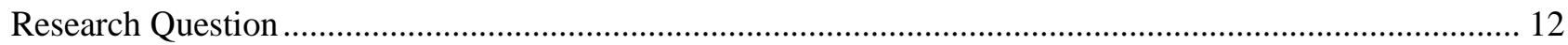



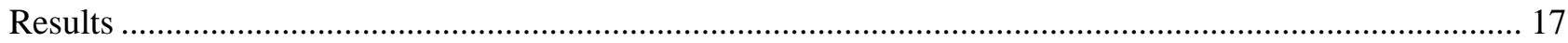

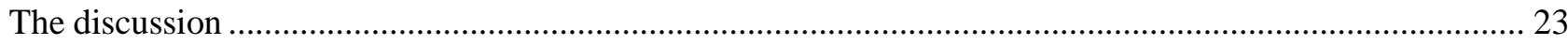

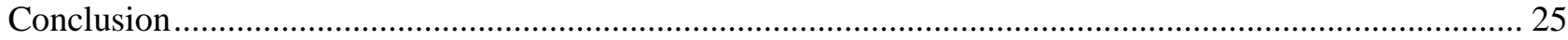




\section{Introduction}

Our challenge is to empower the designer with an impactful immersive tool, enabling the understanding of the design problems' context and the prediction of the future products' nature. By observing designers we have identified their needs in term of tools. An exhaustive study of the virtual reality techniques has showed the opportunities offered by these technologies. The synthesis of this knowledge has taken us to the tool proposition we detail in this paper. We will also explain how we have developed the tool itself as well as how we have evaluated it in comparison of traditional the traditional methods used by industrial designers. In order to achieve this we will first present the industrial context of the study. We will then present our state of the art focused on Industrial design and virtual reality and then expose and explain our research question. This part will be follow by the presentation of our hypothesis, the explication of the experimental setup and the result we achieved. We will then discuss those results and conclude our paper.

\section{From inspiration to innovation}

Modern design studios are aiming to propose innovative ideas: Observing our behaviors and desires, creating connections between dreams, imagining the incarnation of functions and systems, drawing our future. The innovation drives and is driven by the human evolution. An industrial designer must be mindful of his products' environment and end-users but also be able to produce a design breakthrough enlarging the existing product landscape frame. Recent research has established the impact of a ground-breaking inspirational process on the industrial designer concept proposals. Although important research has been executed to understand the industrial designer's inspirational process, serendipity is yet to be understood. To guarantee an evolution of industrial towards the production innovative products, one must understand the anchoring of the concept generation process. Thus we have chosen to study and augment the early design process in order to propose new methods and tools that stimulates and expand the industrial design process.

\section{State of the Art}

\section{Introduction}

In this first section is exposed a knowledge set necessary to the extensive understanding of this paper. We will first detail our vision of design, the macro models and product design methods we refer to. To understand the design activity, we have studied the emotional and decisional processes and their relationship intrinsic to product design. To close the design related chapter, we will present a description of the inspirational methods, tools and process taking place during the early phases of design. Finally we will expose the models on which we base ourselves to understand virtual reality and present an overview of its use in design. This two part synopsis gives us access to the ground rules of designing an inspirational early design tools using virtual reality as a foundation. 


\section{Industrial Design}

Different teams and expertise take part in the complex product design process. Industrial design, Engineering, Sales, Marketing, Management and many more will take decisions and influence the final artifact. We find that timewise, this vision implies that the initial decisions made by the design team influence the structuring and anchoring of the actions taken after the design process. This justifies the high impact of the design team on the final products' morphologies and the user experiments. It also shows the importance of using adequate tools and methodologies during this phase. Our research goal is to ensure that tools used during the design phase are up-to-date and coherent with the designers needs. The next paragraph details our view of the design activity and specifies the time laps we focus our study on.

The success of an industrial design product is not a coincidence. A well designed product has been conceived and carefully studied to correctly fit the market. In order to do this, the designer must gather information from his environment to inspire his creative process and create an artifact corresponding to a given lifestyle, ambiance, atmosphere (Caron, 2005).

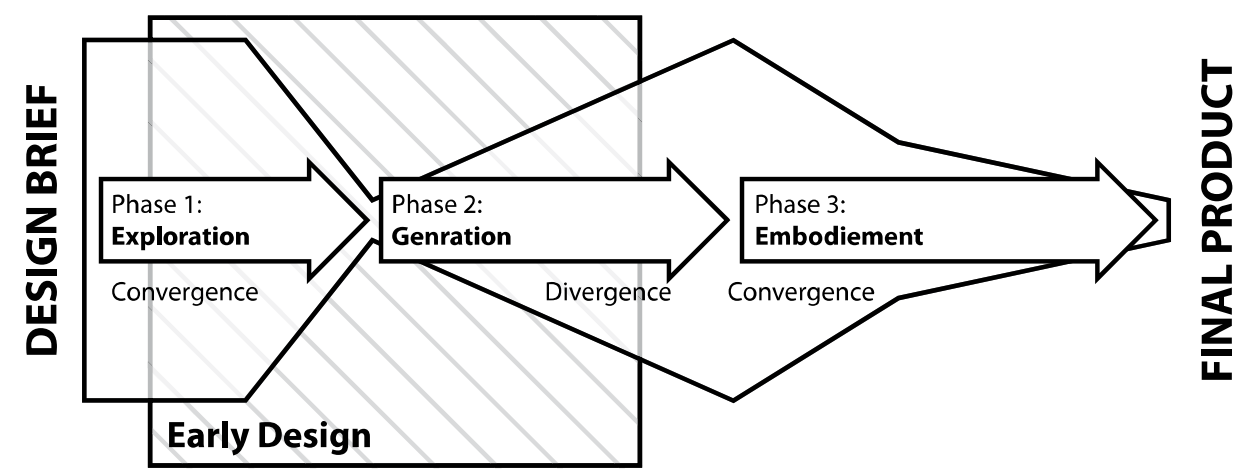

Figure.1 Design process from end to end - Boundaries of early design

We call this gathering the "exploration phase" (cf.fig1 Phase1). It is followed by the "generation phase" (cf.fig1 Phase2) in which the designers will construct formal representations of the product. We have chosen to focus our research on the early phase of the design process as it highly defines the nature of the final product.

Conjoint Trend Analysis is a strategic tool for trend identification. It enables the designer to specify interesting trends by gathering sociological data and contemporary graphical representations (fashion; architectural; design...) (Bouchard, 2005). When he/she processes these pieces of information, he/she generates trend knowledge. Only then can this trend be depicted on a moodboard. And project direction can then be validated or not, with his/her co-workers and clients. The general goal of this exploration phase (1) is to discover trails and spot emerging trends. It also helps the industrial design team in their construction by providing additional information. Design briefs, given by clients, are too often insufficient to start an innovative product design project (3P Project, 2011, Interview designer Bic). The next paragraph depicts how the lack of information necessary to build a concept is burdensome since during early design the parameters the designers 
work with are of an abstract nature.

In the field of industrial design, we are presently encountering a major orientation. Studying the pleasure in acquiring and experiencing a product takes a larger part in Design process. Since the early 1980s' functionalism is gradually being replaced by a more sensorial, emotional and hedonic take on product design (Childs, 2004). The new considerations are more abstract than the primal object parameters (form, color, texture). Nowadays researchers have been developing methodologies on how to design a human/object interaction (Ludden, 2011), emotional impact (Norman, 2002) (Nagamachi M. , 2002) and sensorial relationship (Bassereau, 2001) (Bonnamy, 2008). Designing attractive products therefore calls for knowledge about the sensation, feelings and impressions the products evokes (Schütte, Affective meaning: The Kansei Engineering approach, 2008)and this as soon as possible in the design process. We have categorized these characteristics as high level product parameters in opposition to the low level characteristics being form, color and texture. (Mougenot, 2008) (Kim, 2011). How can the designer operate these abstract, high level characteristics?

A promising methodology created in Japan mentions an original way of seeing user centered design. In this particular vision, the link between a human being and an object is advanced in terms of designing for emotional impact. This link is referred to as 'kansei'. We will now define this term, expose the different methodologies of kansei design and show how these methods could help us enhance the design exploration phases. The term kansei has been introduced from Japanese culture. As it has no direct translation, contemporary researches have been made on the term signification itself. Here are a few of the most referred to definitions: kansei is to be understood as a global and immediate perception of a person and its psycho-physiological impact (emotion, sensation...). In other words kansei is the global feeling one has once he/she has perceived an object or environment through his/her sensory system. (Lévy, 2008) (Harada, 1998). Kansei is the sensory and cognitive effect of an object, environment or situation on a person (Schütte, Engineering emotional value in Product Design, 2005). Kansei is sensibility, signification, feelings, aesthetics, emotion, affect and intuition (Nagamachi M. , 2002).

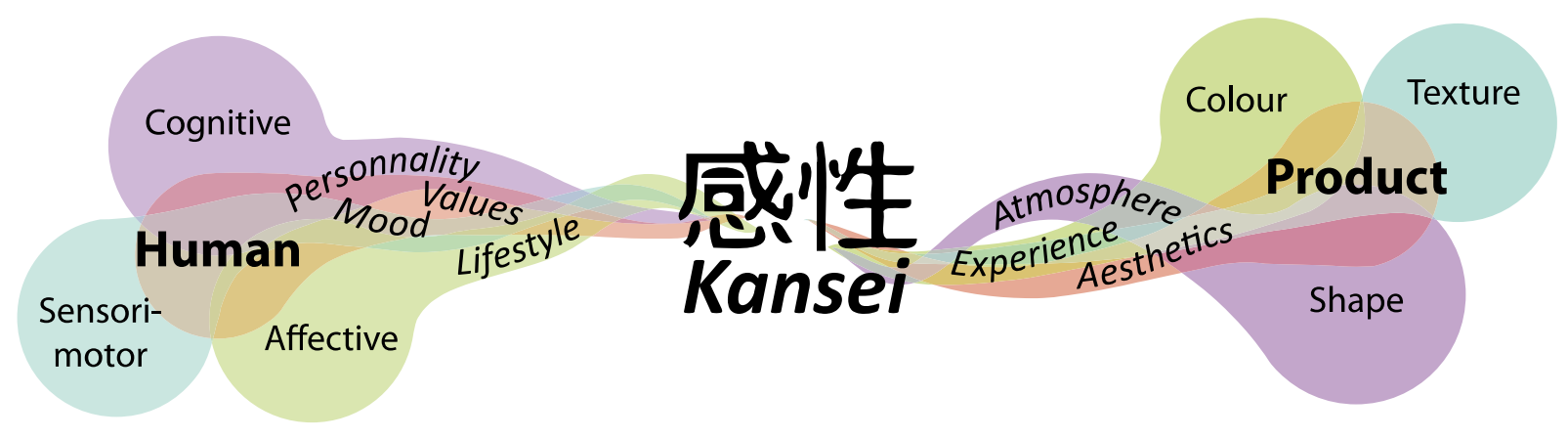

Figure.2 Kansei: a holistic approach of the human/product relationship

The usefulness of the term kansei lies in the holistic approach of a human/object relationship. The duality between Chisei and Kansei evokes the description of the aesthetic judgment by Uhlmann 
(Uhlmann, 2008) : as the correctness of a product depends more of a Chisei matter (cf.fig3) the appeal of a product is closer to the kansei domain. Kansei design is a methodology that centers product design on kansei analysis. Obviously if one understands the desired kansei relationship between the user and his product, one is able to define design specifications that will produce the desired emotions. Kansei design connects specific populations' affective needs with precise products properties. The next paragraph introduces how emotion and decisions are highly interrelated during the activity of design.

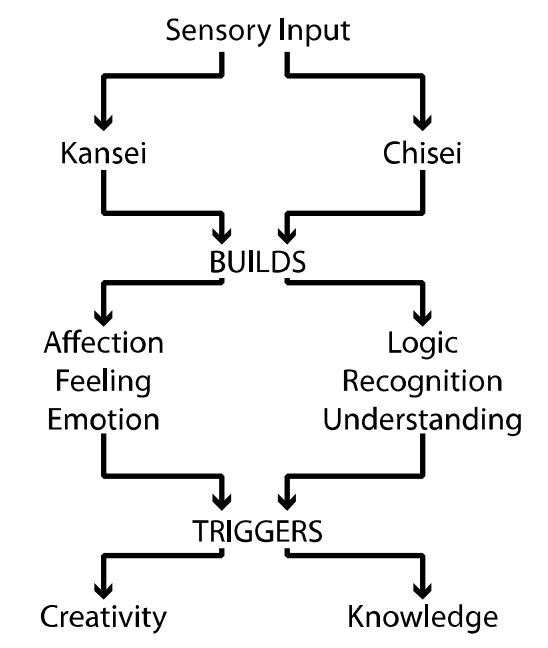

Figure.3 Kansei \& Chisei (Lee et al. 2002)

Our study requires that we understand the connections between the designer and his environment. Next is a classification of the information necessary to the depiction of a perceptual, cognitive and emotional model for the designer. A contemporary theory (Damasio, 2005) regards emotion and cognition as two simultaneous interrelated processes which each take an essential place in the decision process. For instance, the birth of an emotion is the superposition of limbic (emotional) representation of an observed object and the neocortical (rational) representation of the same object. Applied to industrial design, this analysis strongly echoes into Donald Norman's work (Norman D. , 1988): the limbic path being highly linked with visceral level and the neocortical with the reflexive level. From a synthesis of psychological, neurological, behavioral and physiological models we have erected a model of the designer. Our future argumentation development will mainly be based on this model. As the human/object relationship is difficult to apprehend with metrics, we will analyze responses of many natures to a given stimulus, and evaluate the relationship by combining these different measures (Pavlov, 1927). This logic combination allows to bypass the complexity of control mechanism of human reaction. (Reuchlin, 1997) 




Figure.4 Perceptual, cognitive and emotional model

A stimulus occurs when the environment changes from an initial state to another. The constant dual conscious and unconscious analysis of our environment constitutes the decision we take (Pons, 2010), decision about the possible operations and anticipated result (Uhlmann, 2008):

Throughout the industrial design process, the designer assesses his work and his potential inspiration material. This assessment is part of a cycle use by the designer to solve his design problem:

Assessment of an input status with regard to correctness and appeal (bipolarity). This input usually is the output of the previous design action.

Mental anticipation of a new status based on the previous evaluation and anticipation of operations that could invoke this new status.

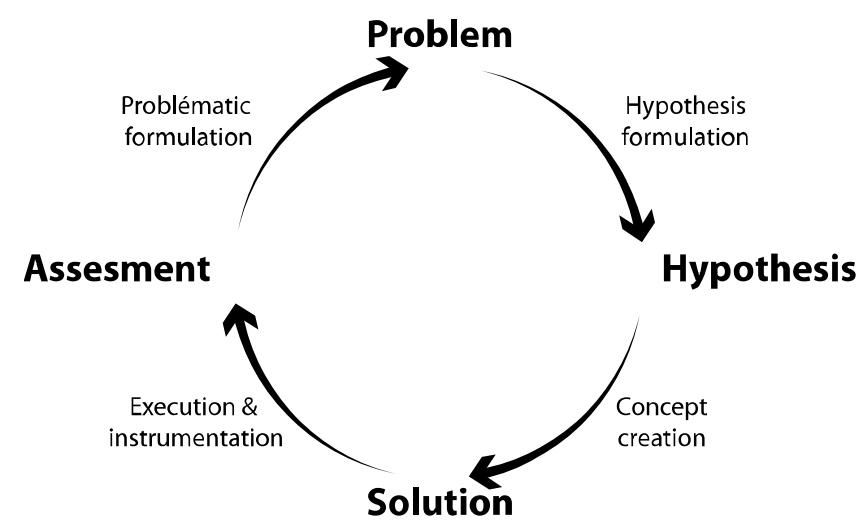

Figure.5 Design Hypothesis formulation and correction cycle (Christofol, 1995)

Aesthetic judgment in design is an objective and subjective process. Objective judgment 
relates to erected rules such as gelstat theory (Wertheimer, 1912) or the chromatic circle of Isaac Newton. It is a calculated logical reasoning to determine if an object representation or his design attributes are acceptable. Whereas subjective judgment is highly linked to the emotional and memory center of the brain and isn't always conscious (Woelfel, 2010). As suggested before and in reference to Damasio's studies, both parts of the judgment (logical and emotional) are quasi-simultaneous and condition each another. This psychological view over industrial design gives us the theoretical basis to understand how information is processed during the assessment phases (cf.Figure5). To assist the designer in early decision-making, tools such as moodboard can be used.

As said before, it is of the industrial designer's responsibility to explore the relationship between the future user and the designed product. Depending on the enterprise culture, project timeframe and willingness of the designers, a moodboard is composed for each trend. A moodboard is a graphical/semantic composition explaining the designers' take on the client requested universe, theme or ambiance. The moodboard has many inspirational functions, such as: the definition of a design context, the trigger of idea generation, the structuring of anchors for mental representations. It constitutes also a formal vocabulary basis for communicating innovating ideas (Eckert, 2004). In other words, this process has a great impact on the success or fail of the designed product. The more the targeted trend is understood, the more the designer will benefit from the information and will be precise and creative in its work. Creating a moodboard isn't unconditional, the synthesis work and stylistic choices can be informally executed by the design team. As the decisions taken during such early design phases represent most of what the product will be, the tools used during these phases must be highly effective. As eighty percent of the design process are determined during the beginning, preliminary analysis and evaluations are the bottleneck of the design process. The stylistic decisions taken during the exploration phase (Phase 1) will freeze a certain number of the designed product parameters.

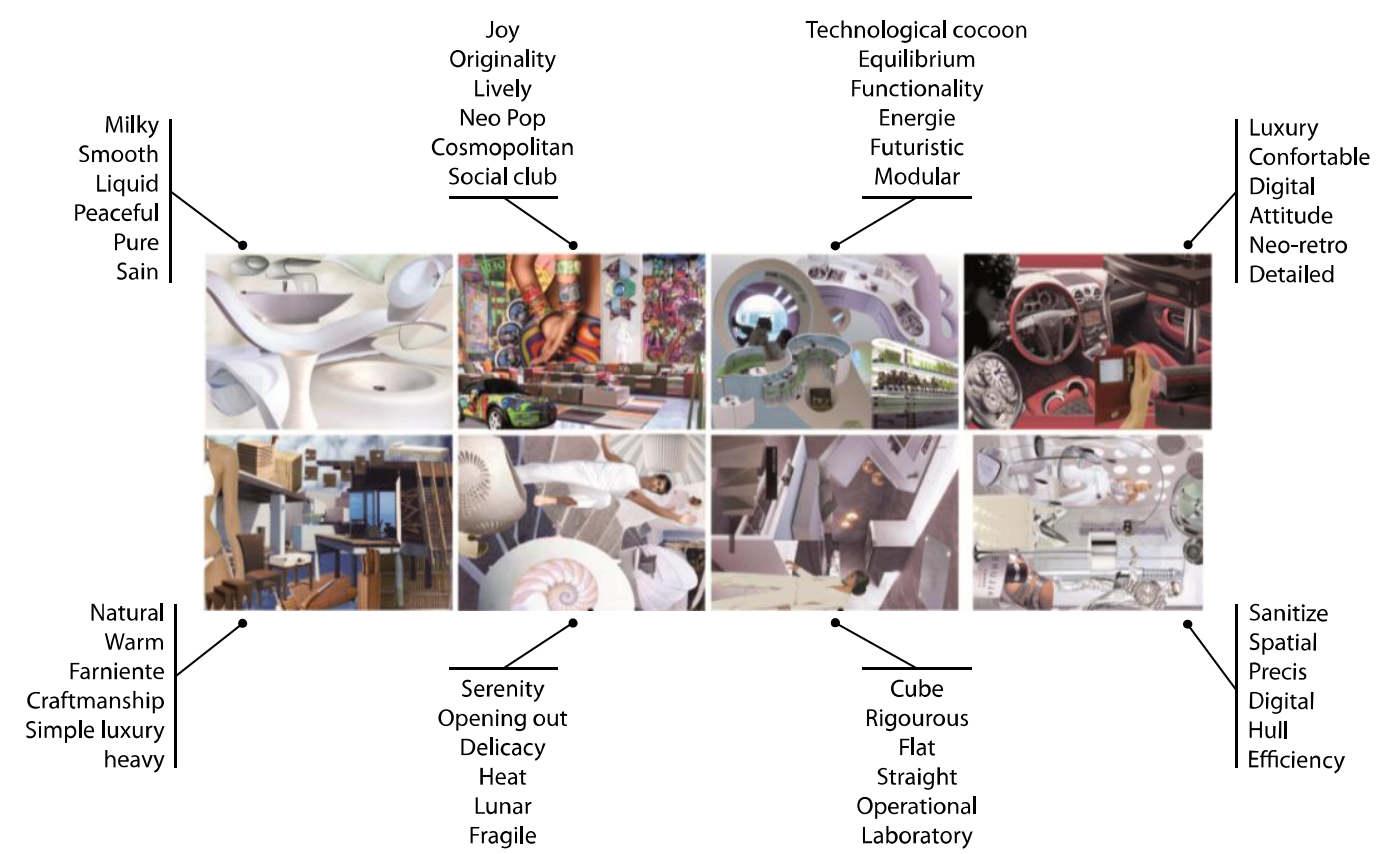




\section{Figure.6 Eight trends identified through the Conjoint Trend Analysis}

In conclusion, the industrial design process is paved with hypothesis and practical decisions regarding how the industrial designer imagines and represents the future product. The product is iteratively generated through decisions based on inspirational material. As we know the emotional brain is involved during the decisions process, thus an early design inspirational tool must engage and emotionally impact the industrial designer. Such a tool would guaranty fidelity in early decisions.

\section{Virtual Reality}

Another large orientation in industrial design is the computation of the design process in search of digital tool creation. Digital tools have been used in many fields to reduce development time, cost or to insure a low error, high quality process (Kim, 2011) (Bouchard, 2003) (Restrepo, 2004). At the moment, they are not highly present in the early design process but rather more used in the detailed design phases. However, at the crossing between industrial design and innovative digital tools, we find virtual reality aided design.

Throughout this paper the term "Virtual Reality" is to be understood in the Fuchs and al. way:

"Virtual reality enables one or more persons to experience a sensor-motor and cognitive [and emotional] activity in a digitally created artificial world. This world can be imaginary, symbolic or a simulation of the real word." (Fuchs G. M., 2006).

We consider a virtual system is qualified as immersive when the user accesses a new motor and perceptual field enabled by a technical device. Immersion can induce the feeling of presence. To be present into a certain space is simply to localize events and objects from a point of view belonging to this specific space as the real/technical device rendering the new reality is occluded. It is now becoming common to encounter innovative products linked to virtual reality on daily basis. Remote control device with motion tracking features, three dimensional movie experiences via stereoscopic glasses, augmented reality on cellular phones... and many more application of virtual reality emerge in every day's life. The standard modern user is ready to accept immersive interactions, and is often fond of them. In recent researches combining designers with mixed reality, participants showed enthusiastic comportment towards the use of virtual tools (Lucero, 2005). This echoes in the virtual reality domain of Serious Gaming. Serious Gaming is a pedagogical tool using videogame strategies to teach or communicate a message (Alvarez, 2007). As the society evolves, virtuality takes a bigger part in our everyday life. Evolved man/machine interfaces such as affective computing (Batliner, 2006) or bodily engagement are more easily accepted. These are satisfying circumstances to propose a new vision of industrial design in which designer and future products interact through digital worlds. Virtual reality is not an end in itself but it is a technological opportunity to answer the designers' needs for new tools.

Virtual Reality Aided Design techniques are already used in some industrially leading Corporation. Nowadays the major function of V.R is to validate 1:1 scale products by real-time 
interaction and replace real models to reduce the projects time and cost. Further than validation, V.R is also used as a virtual support for product generation in engineering design. Unfortunately, the more the activity encloses early stage design, like conceptual design, the less V.R tools are developed and used. A few projects have been exploring three dimensional sketching or virtual kansei based conception (see§ Industrial Design): Sketch Furniture (Front Design, 2006), Tagged in motion (Jung von Matt, 2008), Housemall project (Shibata, 2003), Ilovesketch (Bae, 2009). These approaches tend to explore mechanisms resembling early stages of industrial design. Nevertheless we have observed that virtual reality is not very implied in the first steps of industrial design. Woelfel (Woelfel, 2010) explain this absence in the fuzzy front end of the new product development by comparing the fuzziness of sketching and the geometrical strictness of virtual model data (Buxton, 2007). The necessity of ambiguous non-restrictive representation techniques in the early phases can be the cause of the virtual technologies rejection. Fuchs reminds us that virtual reality techniques can be used to emulate aspects of the real world, but that it can also depict a symbolic unrealistic world.

"Virtual reality has the potential to go farther than copy $[\ldots]$ and to generate imaginary environments out of an artist's or a science fiction writer. In this case the environment isn't necessarily a simulation of the real world, particularly concerning virtual entities laws" (Fuchs G. M., 2006)

Aiming at a numeric design process can also create a dependency to computerized system and reduce abstraction capacities (Dorta, 2001). If simulation is a cheap and quick way to evaluate a product design, it is fundamentally different from reality. The perception of an object can therefore be distorted. For this reason, virtual reality cannot be the only representation type used during the new product development (Kadri, 2007).

In conclusion, the use of virtual reality techniques occurs scarcely in the early steps of the design process although it seems that the technological possibilities could enable preliminary design studies. The sensorial interaction power necessary to an early industrial product evaluation is available. And the possibilities of keeping the fuzziness required in premature representation can be obtained in three-dimensional interactive environments.

\section{Statements: Limitations in Early Intermediate Representation}

Intermediate representations are the externalized traces of the design thinking. They represent the product as the design process is executed. Intermediate representations are used for product evaluation, to identify defects, find alternative solutions, estimate fabrication time/costs and stimulate designer/designer or designer/client dialogue (Göbel, 1994). They allow simulating the essential properties of the product and are used from the first ideas to the final artifact rendering. During the design process, hypothesis are made and evaluated by means of intermediate representation. These representations are often hyper-centered on visual evaluation. As the fuzzy front end of design goes, hypothesis made in this phase concern many of the sensorial modalities (Bassereau, 2001) (Guenand, 2008). It is difficult for a designer to evaluate future products 
properties when unable to appreciate them under their multimodal form (Kadri, 2007). A design defect can even only be detected once the final prototype is rendered and subsequently release additional funds or mobilize a design team for an undetermined time. Thus it is crucial to identify any possible defect or promising designs through early representation as soon as possible to ensure a successful and competitive product. Traditionally and in an orderly fashion, the different intermediate representations are: Moodboard, Drawings, Digital Mockups and Physical Mockups.

The design process is dotted with intermediate representations to help evaluation and decision making. However these representations have limitations and embody only part of the design issues. The more the representation simulates the final product, the better the represented solution can be evaluated, the better the strategic decisions concerning the object design can be trusted (Kadri, 2007). In paradox, these representations need to be ambiguous so that they leave space for design thinking. As these representations are supports for exploration, they are required to be unfinished and with a certain degree of abstraction (Buxton, 2007). To sum up, a good design intermediate representation has a good balance between the information it conveys and the questions it asks according to its time framing in the design process. Concept and knowledge theory identifies these spaces as "conceptual space" (questions, expands) and "knowledge space" (answers, sums up) (Hatchuel, 2009). Representations found in the early design process are more of a conceptual nature in opposition to late design representations which are proposals.

The designing personnel may rapidly experience the future product through $2 \mathrm{D}$ techniques such as moodboards or drawings (Won, 2001) and as said before, this medium gives the designer the possibility to think over and into the gaps of his fuzzy representations. On the other hand this medium gives no dynamic or interactive possibilities; it only gives the option to explore the object or tread through a fixed point of view. Numeric data and representation enables a certain liberty on parameter modulation. For instance a color parameter can easily be changed on a digital support. Another limitation to the 2D representation is the lack of sensorial modality allowed by this type of representation. The 2D means does not permit complex sensorial interaction. For example, the moodboard collages only simulates an atmosphere using the visual modality (Bassereau, 2001) Visuals evaluation mode has a wide monopole over the early intermediate representations. Few are the haptic, olfactory, auditory... rapid representation made available to the designing team in order to evaluate what can emanate from the future product. Vividness is the power with which an object imposes itself to consciousness via emotional images. A vivid message gets more attention because of the intense emotions it evokes. (Kelley, 1989). A vivid media would exhibits the appearance of life. 


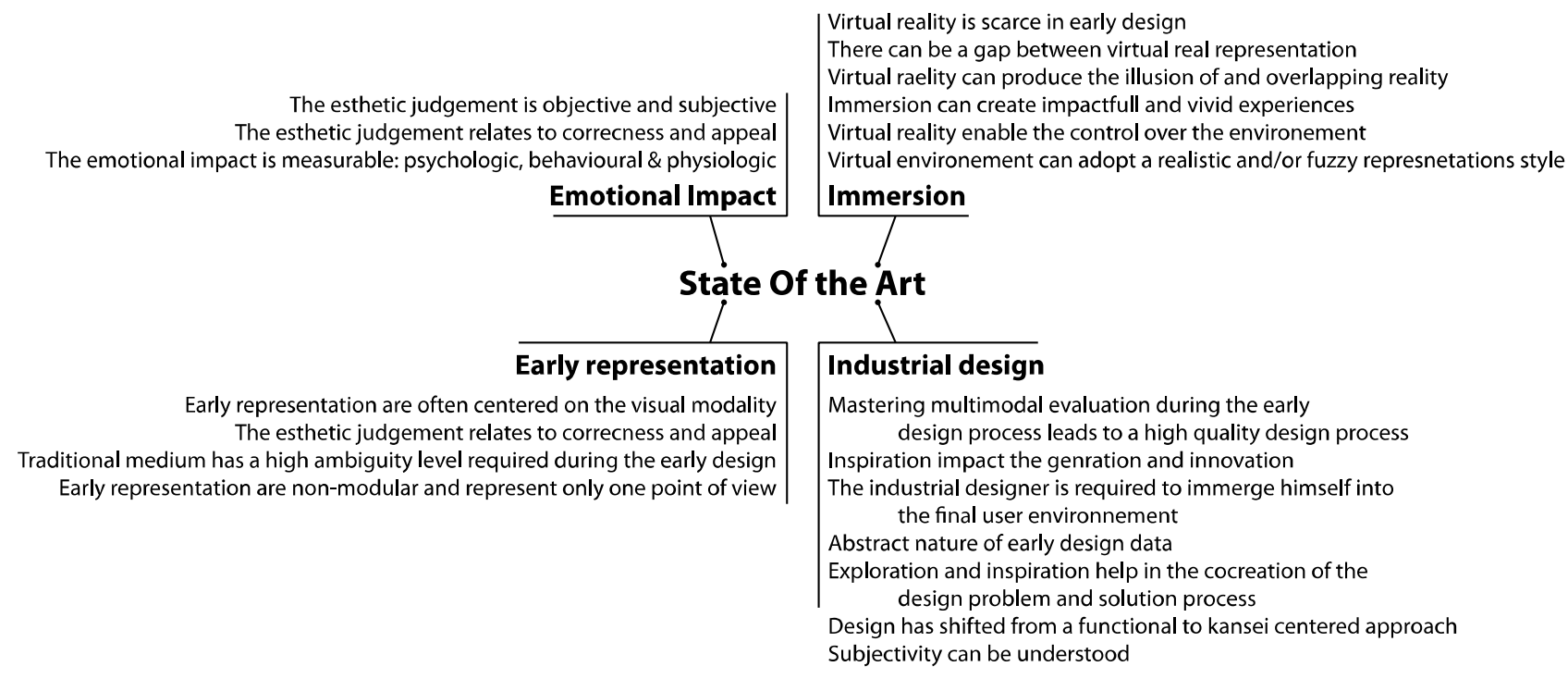

Figure.7 Statement synthesis

To summarize, intermediate representation are a formal hypothesis generation and evaluation tool. They enable the identification of defects and assets as well as open the dialogue over design attributes. In the contemporary design process, the intermediate representations are highly visual. Time wise, the more the design process advances the more precise design attributes are represented. During early design, working on the products' kansei implies a media that enables a good apprehension of the emotional aspect of a trend and enables the represented trend to appear vivid or as being part of the designers world.

\section{Research Question}

As we have pointed out earlier, the designer needs to investigate and define the future products kansei as soon as possible. Moodboards are the first synthesized representations of the product and are traditionally the first kansei assessment tools. They assist the designer in the validation of a specific design attributes and of an overall style and direction for the project.

How can kansei exploration be augmented by virtual reality techniques during early design phases?

\section{Hypothesis}

This question supposes the design of an innovative appropriate early representation enabling early kansei identification. This representation needs to convey a high kansei impact on the user, appeal to the designers' affect, and inform the designer of the interesting trends and of the high-level and low-level design attributes. At the same time, it should conserve a good abstraction level to give way to a fertile concept generation phase.

H1: Our hypothesis is that the spatialization of trend data changes the kansei of the trend. 
As far as representing an ambiance, a lifestyle or a trend, a two dimensional media is not the most impactful media available. We propose to spatialize the trend data in an immersive three dimensional environment. By adding a third depth to moodboards in respect to the initial moodboards composition, we have created a three dimensional trend environment. Thus keeping the immersive moodboard a scene, as was the traditional moodboard, but adding interaction between the designer and the trend by combining the designer and confronting the designer to the early representations reality.



Figure.8 Moodboard spatialization

The virtual reality space is collocated with the real space and a motion parallax renders the experience of this 3D trend environment logical, as the perspective is recalculated in real time. The $3 \mathrm{D}$ trends are retro projected on the stereoscopic screen which acts as a window from the real environment to the virtual trend environment. What originally was a static picture becomes a set of $3 \mathrm{D}$ billboards participating in the illusion of a vivid and plausible world. While the perspective is modified according to the designer's eye position and the rules of realistic perspectives, the individual perspectives of each billboard picture give an abstract surrealist feel to these worlds. Using two dimensional images as raw material for the construction of these virtual environments contributes in keeping this representation a fast and simple tool. The depth axis augments the space available for trend information; it also enables the designer to see behind an object and bypass occlusion. In this way the designer is also able to combine different objects in its immediate eyesight by simply walking from a point to another in the real world. The next chapters will relate how this new media impacts the relationship between the designer and the trend with an insight on the scientific methods and protocols we chose to apply.

\section{Experimentation}

\section{Objective \& Method}

To understand how the early activity of industrial design can be modified by virtual reality techniques, we have first decided to observe the human/trend relationship. With equal content, what is the impact of immersive media on a trends' kansei? We have constructed a two condition 
comparative study in which a set of twenty designers are shown two immersive moodboards (IM) and two traditional moodboards (TM). They are asked to fill a series of questionnaire concerning their experience. The traditional and immersive moodboards are both shown on the same screen and in the same format (height $180 \mathrm{~mm} x$ length $240 \mathrm{~mm}$ ) to subtract any bias due to the display size. Our objective is to isolate the spatialization condition and analyses its impact on the inspirational trends' kansei.

As affect, feelings and emotions are so volatile and not directly observable objects, we have developed a method to objectively measure the designers' experience. We observe responses to controlled stimulus throughout our experimentations. To understand kansei and to triangulate it, we base our studies on the analysis of physiological, cognitive and behavioral cluster of measured data. By measuring these various aspects of the human being, it is easier to reach a conclusion concerning the observed phenomenon between stimulation and response. For instance a phenomenon visibility is reinforced when two of the measures echo themselves with a strong correlation. This helps bypassing the inbuilt human reaction control mechanism. By combining these different data sets, new meaning can be unveiled. To model the emotional arousal response to a stimulus in a physiological way, we have chosen to use Galvanic Skin Reponses which is an interesting measurement owing to its advantages, including low price, simplicity of use, high sensibility, and reliability. It provides continuous information and detects very sensitive amount of arousal (Kim, 2011).

The cognitive response to the stimulation is measured via semantic differential questionnaires (Osgood, 1957) (Likert, 1932) (Mantelet, 2006). The first questionnaire is a set of ten opposed semantic descriptors (we shall call them 'couples of descriptors') per moodboard, obtained via precedent tests through designers' verbalization, selection and sorting. We use them to understand the semantics restored by a moodboard to a designer. The next questionnaire is a set of 10 emotional terms per moodboard, obtained via precedent tests through selection of terms in an extensive list (Sacharin, 2010). Via this questionnaire, designers are also asked to evaluate the valence and intensity of their emotional state when stimulated by trend representation (Lang, 1997). As it is likely the immersive and traditional moodboards only induce secondary emotions during cognitive emotional evaluation, we have left out the study of dominance often used to assess primary emotions (Mantelet, 2006) (Damasio, 2005) (Ekkert, 2008).

Behaviors are registered through two major measures: Eye tracking and variation of point of view or head position in space. When facing a stimulus, the designer is equipped with an eye tracking helmet indicating where his gaze position is. As three dimensional perceptions are not only due to stereoscopic view but also to movement in space, the movement is also an indicator of the users' engagement and desire to experience the virtual reality.

Here is an exhaustive list of combinations made to understand kansei: The combination between emotional questionnaires $(\psi)$ and galvanic skin response $(\varphi)$ may confirm the felt emotions of the designer panel. Pairing emotional questionnaire and users' movement can be used to 
determine specific behaviors due to spatialization of the data. An indicator of the potential capacity of an object contained in the moodboard to arouse the designer emotion can be created by combining galvanic skin response $(\varphi)$ to gaze position data $(\beta)$. And at last the galvanic skin response $(\varphi)$ can be combined with the designers' point of view position $(\beta)$ to detect and correlation between movement and galvanic skin response.

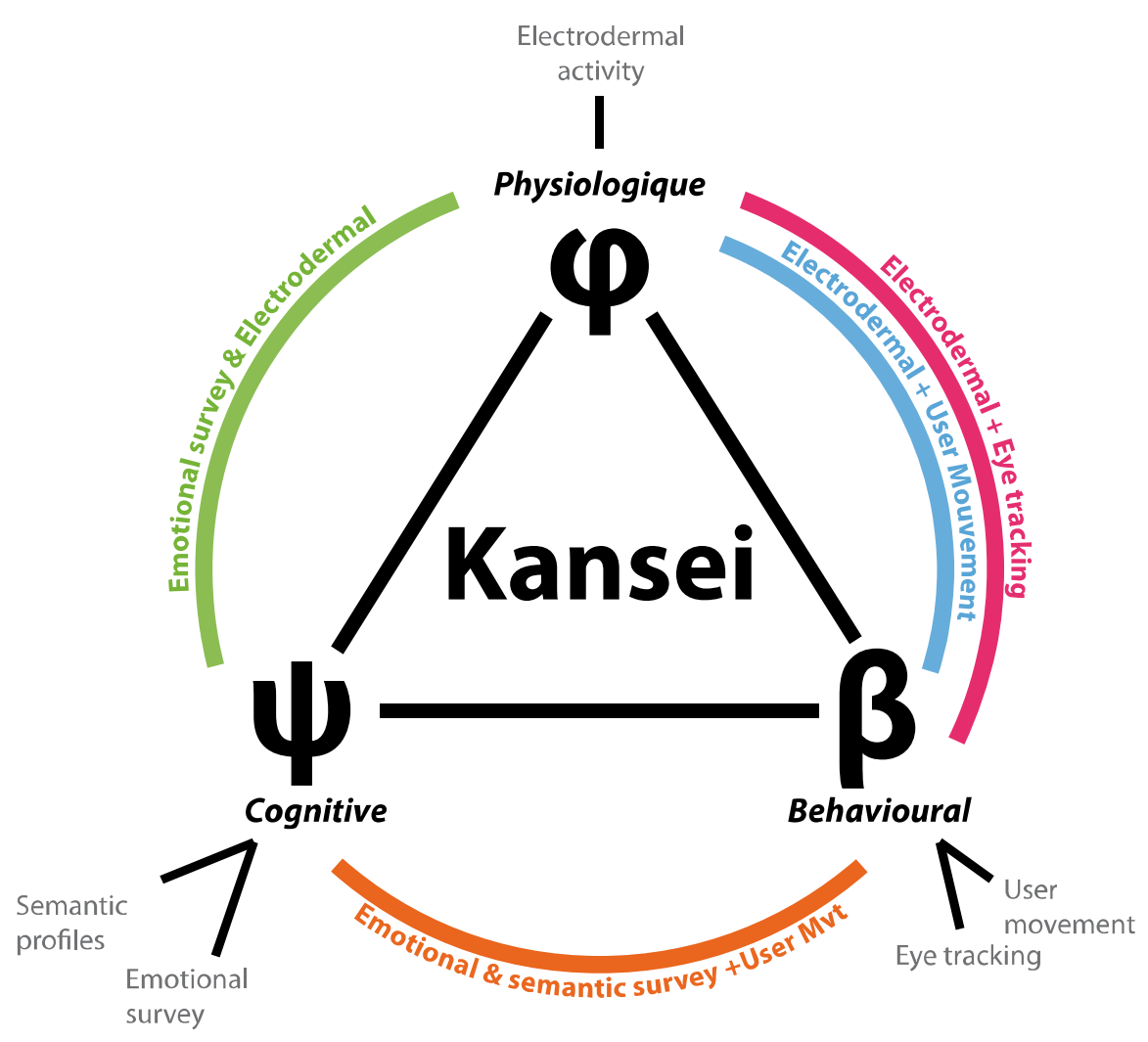

Figure.9 Observed physiological, psychological \& behavioral data cluster

The scheme underneath figure.10 explains the setup of the experimentation. While the designer is stimulated by alternating 20 seconds of immersive and traditional moodboards, his ocular activity is measured with the mono-ocular HED, his galvanic skin response is measured with the amplification unit and a set of two electrodes covered in isotonic recording gel, and his posture is recorded via depth camera and a motion tracking device composed of six infrared cameras paired with constellation of reflector spheres. 


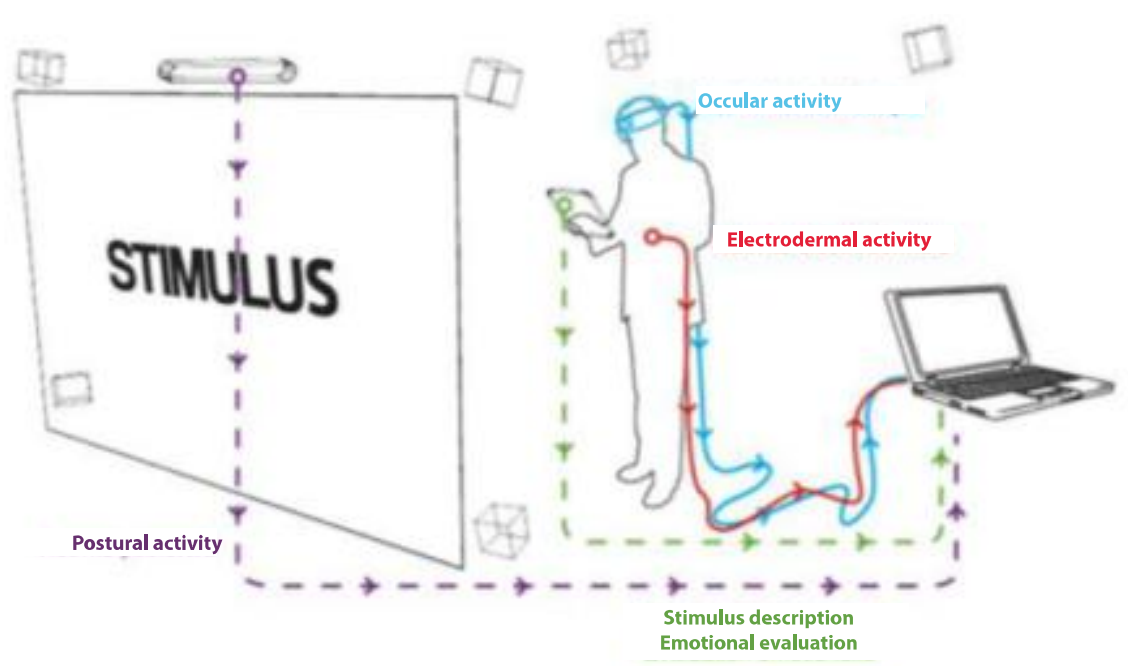

Figure.10 Kansei measurement

After the 20 seconds of passive stimulation, the designer is now allowed to answer the questionnaire displayed on a 9inch touch tablet. This is made so that the cognitive activity of answering the questionnaire does not interfere with the galvanic skin response and eye-tracking recording. Between each stimulation and questionnaire phase, there is a relaxation phase of 60 seconds during which the stimulus is replaced by a neutral slightly off-white gradient in order to stabilize the emotional activity and flat line the electrodermal signal. However it can be 10 to 20 seconds longer if the subject designer isn't calmed by then.

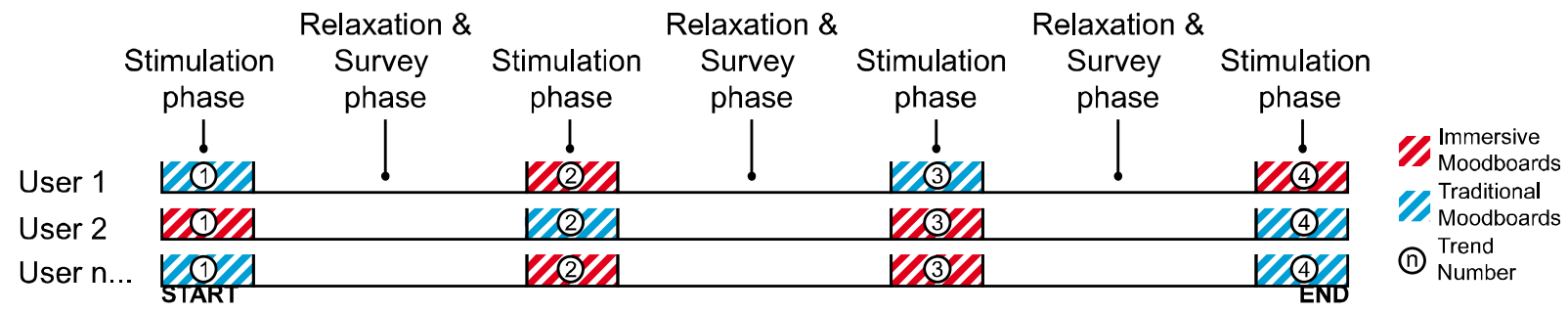

Figure.11 Randomized condition protocol: Immersive / Traditional

Each designer is shown a moodboard and equipped with an eye tracking system. He/she can be it in immersive condition or in non-immersive condition.

This enables comparison of the gaze activity. Four moodboards were chosen out of a panel of ten moodboards as stimulus materials. All moodboards were designed after a method inspired from Conjoint Trend Analysis (Bouchard, 2005). They were chosen by eight $\mathrm{PhD}$ design students who were asked to grade how inspiring these trends were. Only the six moodboards that had the highest inspirational scores were kept. After this first pass, a second selection was made in order to obtain four trends with no apparent kansei similarities to acquire certain representativity over styles, ambiance and trends. 


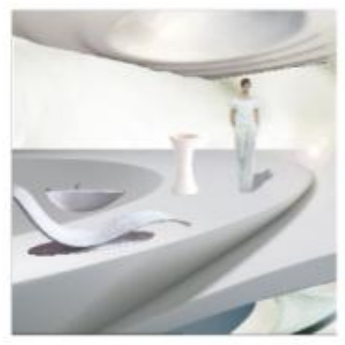

Lactos

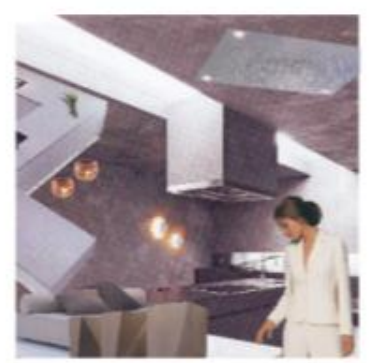

Cube

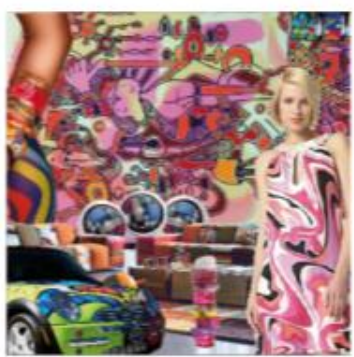

Art \& Fun

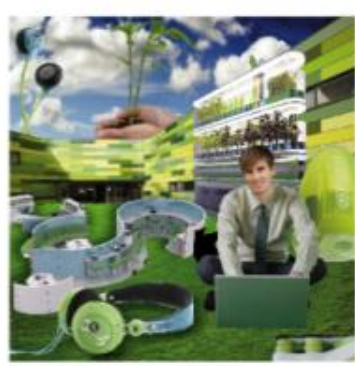

GreenTech

Figure.11 Moodboards selected as stimulus for the experimentation

These moodboards were then spatialized to obtain their virtual reality double. This spatialization was made in respect to overlapping and object to object proportions so that if a designer hypothetically stood perfectly motionless in front of the stimulus, he would observe the same visual whether in immersive or non-immersive condition. Thus we have created two comparable conditions: stimulation of the designer with Immersive Moodboard (IM) in comparison to Traditional Moodboards (TM). One of the intricacies of this study is that we are studying a complex system in which a designer looks at a trend through a support. Deduction on causalities of a certain relationship between designer and trend can be unclear due to the fact that subject, object and media are merged during the experiment. (Bachimont, 2004)

We have categorized the designer population by asking them how frequently they were in contact with virtual reality devices. This expertise is characterized by three levels:

Neophyte: "I've never been in contact with an immersive system before this experimentation" Initiated: "I've been immersed more than three times..."

Expert: "I work with virtual reality"

To spot a potential correlation between the expertise in virtual reality on emotional response, we performed a linear regression study. No apparent statistical effect of the expertise on the physiological emotional response was detected. Designers with a high expertise in virtual reality are as emotionally impacted as neophyte designers. This excludes the possibility that a high emotional activity is due to the first encounter with a virtual reality technologies. However our expert and initiated sample size is small with $60 \%$ neophytes, $25 \%$ initiated and $15 \%$ experts.

\section{Results}

In this section, based on a comparative quantitative and qualitative study protocol, we intended to identify what are the modifications in a moodboards kansei when the contained information is spatialized. The analyses of the results are summarized in the following subsections:

Physiological $(\phi)$ : comparison of electrodermal activity through analysis of emotional peak rate and intensity. 
Cognitive $(\psi)$ : comparison of the semantic and emotional profiles, of the valence and the intensity of the designers' emotional state.

Behavioral ( $\boldsymbol{\beta})$ : Study of the ocular activity $\&$ head position movement.

Physiologic/Cognitive $(\phi / \psi)$ : Data matches between electrodermal activity and emotional questionnaire.

Physiologic/Behavioral $(\phi / \beta)$ : Emotional potential of individual objects, Non-interaction of physical activity on electrodermal measurements.

Cognitive/Behavioral $(\Psi / \beta)$ : Junction of emotional questionnaire data and behaviors in virtual reality

\section{$(\varphi)$ : Comparison of electrodermal activity through analysis of emotional peak rate and intensity.}

When analyzing the impact of virtual media on the emotional physiological response through electrodermal activity, we took particular interest in two specific items: The rate at which a person emits an electrodermal peak and the normalized averaged intensity of the electrodermal activity.



Figure.12 Impact of spatialized immersive media on electrodermal peak rate

The peak rate shown in Figure 13 is much higher in three of the four moodboards comparisons. At the most, the difference of peak per minute is 2.2 points higher in immersive condition for the "Green-tech" moodboard. The peak augmentation phenomenon is less visible on the "Lactos" moodboard. This effect can be caused either by the difference in content, in composition or because of a protocol effect. However we still observe a major tendency for the peak rate to be higher when a designer is confronted to an IM. Only a rapid change in electrodermal activity, larger than the largest difference in conductance fluctuation measured during relaxation phase was considered a peak.

As for intensity in the electrodermal activity, we have shown that the immersive condition 
induces a higher intensity response. These two measures the two graphs on Figure 14 express the same phenomenon, indicating a stronger physiological emotional response while virtually immerged.

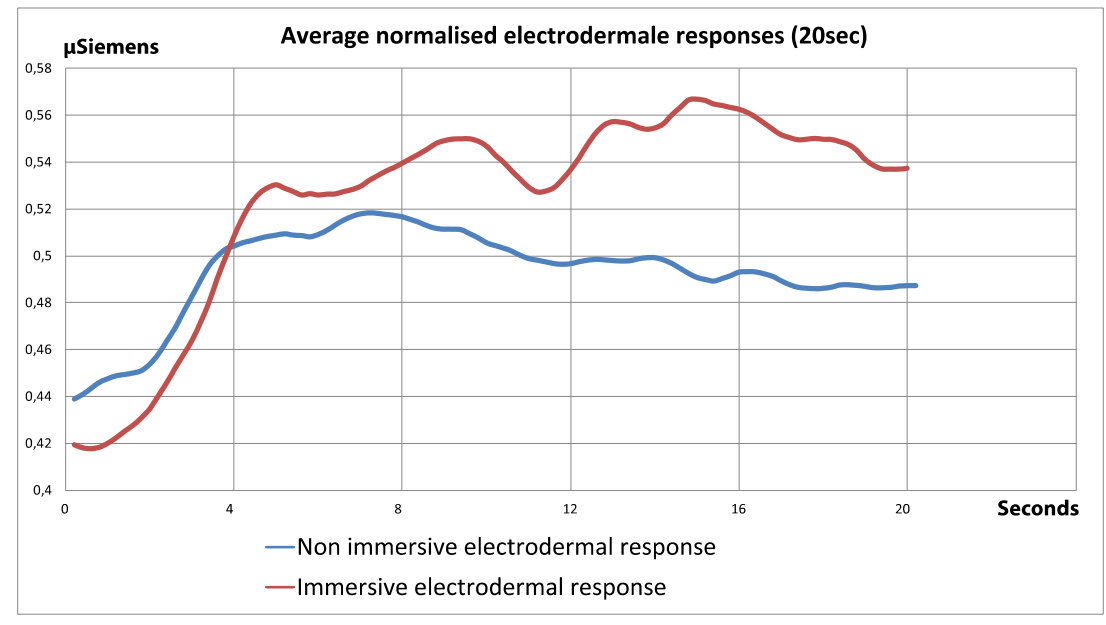

Figure.13 Impact of spatialized immersive media on electrodermal response intensity

Our first observation of the kansei difference tends to show us that the emotional activity of the designer is higher when confronted with immersive version of the moodboard.

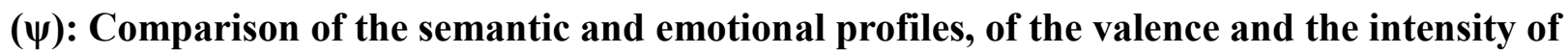
the designers' emotional state.

When comparing the semantic profiles of the two types of moodboards, we focused on specific terms which scored differently when assessed in IM/TM. We also looked at variance of the designers' answers to understand if a certain media induced grouped or spread answers. First of all, on forty couples of descriptors we observe that only a fourth $(10 \%)$ is significantly graded differently from IM to TM. This indicates that both IM and TM are semantically evaluated as close. However we denote a few interesting differences. For instance the couples open/closed; aerated/confined; organized/disorganized all refer to spatial attributes.

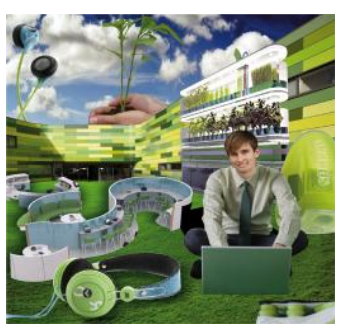

GreenTech

IM TM

Opened Closed

Organized Disorganized

Aired Confined

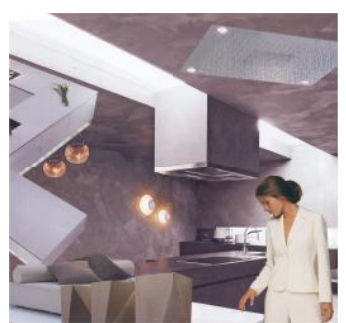

Cube

IM TM

Calm Lively

Futuristic Retro

Conventional Artistic

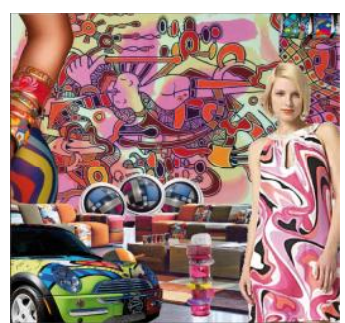

Art \& Fun

IM TM

Elegant Coarse Bright Dark

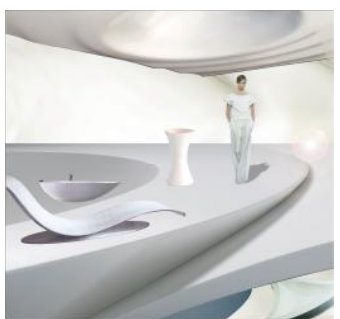

Lactos

IM TM

Beatiful Ugly

Clean Dirty

Figure.14 Difference between IM \& TM semantic profiles

The "GreenTech" IM appears to the designers as open, aerated and organized as the TM is 
more closed, confined and disorganized. These semantic changes seem logical, as the spatial characteristics of a TM are modified to produce an IM. The ambiance and style communicated by the stimulus can also be affected. Still these differences are only a tenth of the total descriptor couples. In average the variance of the designers' answers when referred to an IM are $40 \%$ lower that of the when referred to a TM. This strongly indicates that the potential of an IM to communicate the same semantics to a group of people is higher than that of a TM. In conclusion, these results show that every designer perceive the same semantics; the immersive moodboards helps in the definition of a design context, the structuring of anchors for mental representations and guaranties the use of a formal vocabulary basis for communicating innovating ideas.

In the same way as with the semantic descriptors, we have observed how spatialization of the trend information impacts the way people asses their emotions. The emotions terms are not presented with their opposites: no couples of descriptors for this questionnaire but terms proposed on a one bound scale. Thus when the designers select some terms, we get information not only on the emotions that are felt, but also on the intensity of their feelings. In a similar way, we extracted only the significant differences between the IM and the TM emotional profiles. Here are the results related to this questionnaire study.
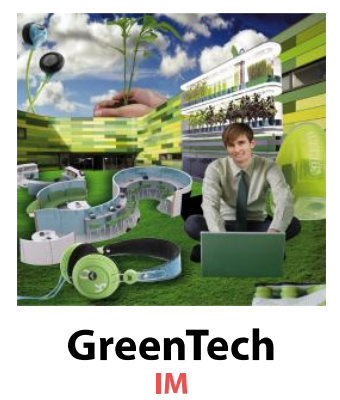

Stimulated Thrilled

TM
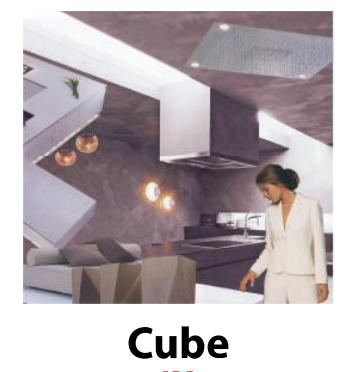

IM

Curious Interested

TM

Relaxed

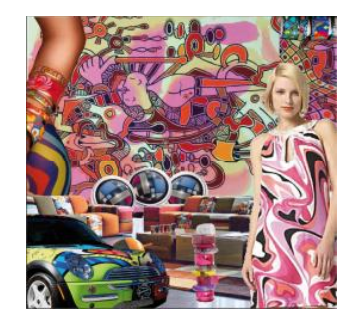

Art \& Fun

IM
Inspired
Interested

TM

Scornfull

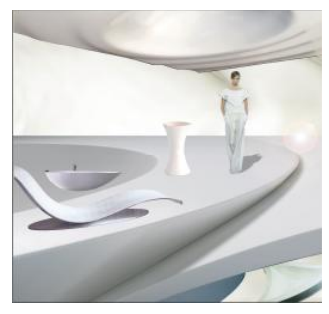

Lactos

IM

Sad

TM

Touched

Figure.15 Difference between IM \& TM emotional profiles

The overall phenomenon shows how more attentive the designers are when they are in front of an IM. Stimulation, thrill, curiosity, interest, inspiration are remarkable virtues for an inspirational representation. Once again when using an IM, the tool experience is better and more appropriate for the inspirational phases of the design process. The interactivity seems to focalize the attention through immersion and playfulness. Seven out of 10 observed differences are in favor of the IM, indicating that the immersive condition induces more intense emotions while appealing to the designers. When interrogated on the valence of their global emotional state, the designers qualified all the IM as inducing a positive emotional state whereas two of the four TM were qualified as negative: spatialization and interactivity turned two trend emotionally negative experiences into emotionally positive experiences. 


\section{( $\beta)$ : Study of the ocular activity. \& movement}

The next result concerns the study of the eye behavior.
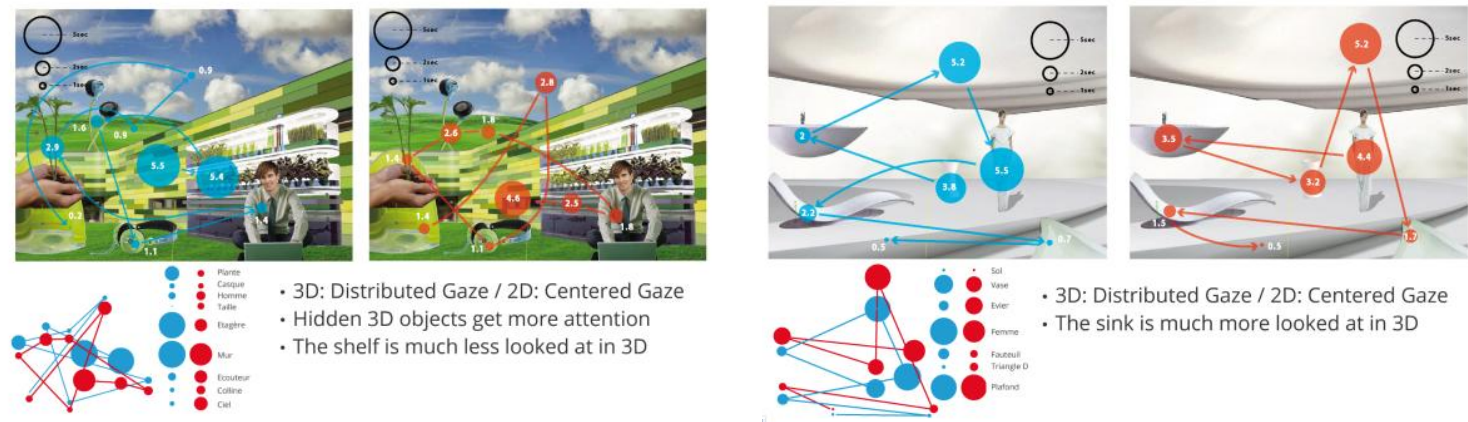

Figure.16 Ocular data comparison: critical trajectories \& total time/object

First of all, it is important to understand that in the IM it is possible to look behind an object. The overall area of a visual is thus bigger than that of a TM. By moving in the interaction space the designers have access to the side and are able to confront two objects that wouldn't have been close in a TM. The first behavioral difference we observe is that the designers have a tendency to distribute their gaze when in immersive condition. The immersive space is visually apprehended in a more global and holistic way. This is confirmed by the time per object measure which indicates how long a designer looks at a particular object depending on its size. Our study shows that designers spend more time looking at a small number of objects when in traditional condition. The moodboard is a stylistic space created by the differences between the objects it is composed with. A media inducing a global approach to style representation should enable a good assessment of the message and meaning of the represented trend.

\section{$(\varphi / \psi)$ : Data matches between electrodermal activity and emotional questionnaire.}

\section{Emotional survey}

- When comparing emotional profiles, $83 \%$ of the emotional terms are said to be felt in a higher intensity in IM in comparison with TM.

- The designers assess the emotional impact of an IM higher than that of a TM.

- When evaluating the valence of the emotional experience: two of the four TM are judged as having a negative impact whereas all the IM have a positive impact on the designers.
Physiological measures

- The physiological emotional response is measured as more intense when in IM.

- Emotional peaks frequencies are higher in IM. 
Figure.1 Recapitulative table of data matches between electrodermal activity and emotional questionnaire Physiological measures and cognitive measures match to show that the emotional impact of a trend is higher when it is spatialized. The tested designers express themselves as being stimulated, thrilled, and interested. This settles the fact that their experience is intense and that they are emotionally engaged in their activity.

\section{$(\varphi / \beta)$ : Emotional impact of individual objects, effect physical activity on electrodermal measurements.}

By combining galvanic skin response and ocular data, we can point out what specific objects are looked at when an electrodermal peak is produced by a designer. On figure 18 is a sample measured on a designer while observing the moodboards. The physiological response to the four moodboards is clearly visible in form of groups of peaks. It is also possible to see the stabilization phases during which the designer is shown a white static image. The red dots correspond to the designer's gaze, caught by a head camera during an electrodermal peak.

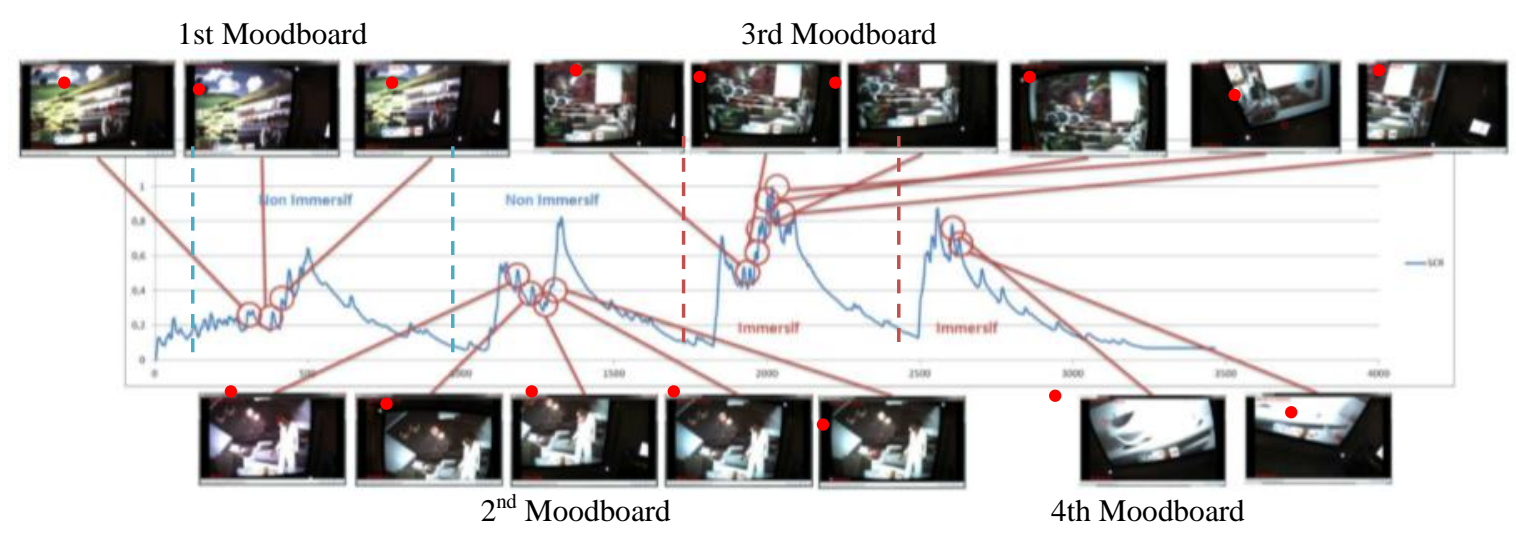

Figure.17 Example of gaze position and galvanic skin response peak combinatory analysis

This combination of measures shows us the physiological emotional reaction during inspiration process and enables us to trace back a peak to a single object in the moodboard composition. By linking peaks to objects it is possible to describe the propensity of an object to generate the highest electrodermal peak emitted during an individual stimulation.

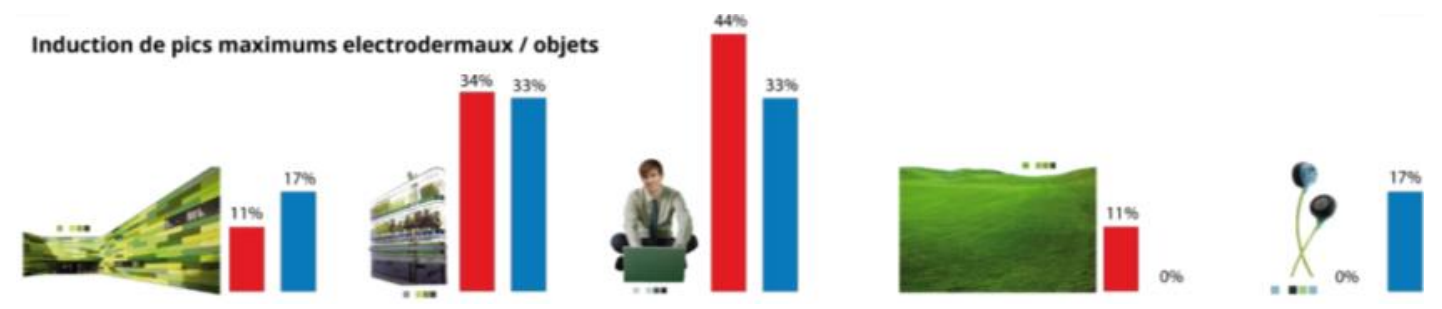

Figure.18 Propensity of maximum peak induction per object depending on media

For instance we observe that the human figure and the shelf have a tendency to generate high intensity peak on the "Greentech" moodboard whether in immersive on traditional condition. For practical reasons we only show the "GreenTech" bar graph, however the human figure are top peak inductor in all the tested moodboards. We suggest this result is the consequences of an empathy 
phenomenon base on mirror neurons. (Rizzolatti et al., 2007) (Wicker et al., 2003). The emotional resonance between moodboard and the industrial designer is crucial during the inspiration phase.

\section{( $\psi / \beta):$ Junction of emotional questionnaire data and behaviors in virtual reality}

We have identified a very common behavior induced by the spatialization of data. By looking at analyzing the gaze and head position, we find that the designers try looking behind objects and try to find the spatial limit of the virtual environment. This resonates with how the designers evaluated themselves: curious and interested. The designers said themselves more thrilled and stimulated in IM; it resonates behaviorally with their head movement quantity, much being higher in IM. Curiosity, stimulation, excitement and interest are all manifest during visual interaction, with movements of the body, such as gaze and changes of head position. Thanks to this multidimensional approach, we have identified the differences between immersive and traditional moodboard kansei.

\section{The discussion}

By adding depth to the moodboard we observe a series of changes in the designer/moodboard relationship. These changes related to kansei are summed up in the next scheme. Immersive moodboards induce:

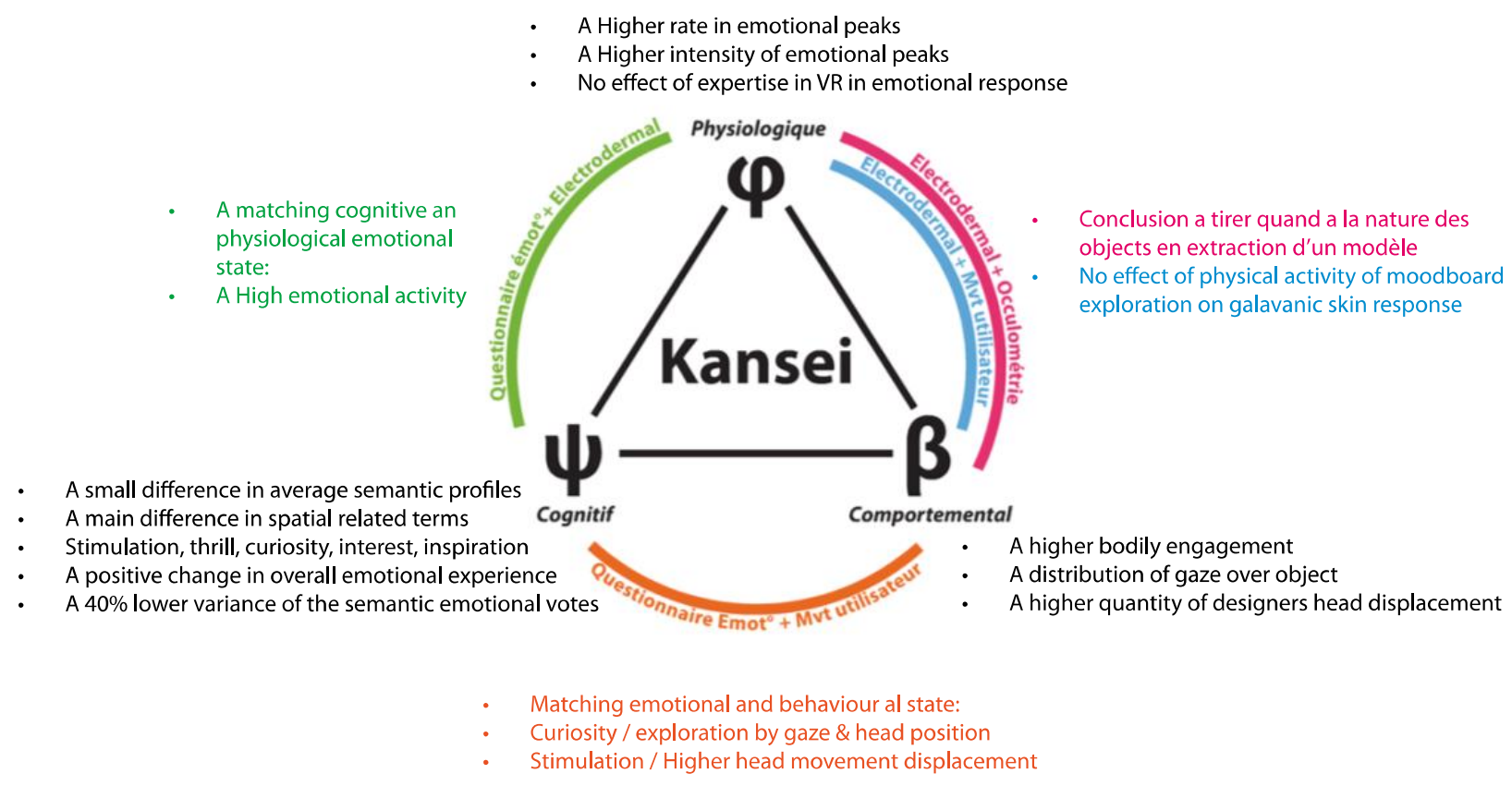

Figure.19 Results and findings

This comparative study observes how a designer reacts when given inspirational trends information through an original structure. To record the kansei dimension of the immersive moodboards we chose to narrow the activity of the designer to a simple contact with trend information. We have thus recorded and analyzed the kansei of a designer looking at a trend and not a designer using a trend as a basis for conceptual proposition. Still the tested populations are trained designers and able to contextualize the moodboards retrospectively to their activity. 
Through our analysis we tag a number of augmentations that impacts the design process. It benefits in different ways of our system and new methodology. This paragraph lists the scientific input of our research and details the impact it has on the design process. Using programmed trend environment enables a series of augmentation:

1. Capitalization data base: Every design taken on the trend representation is recorded and compiled in an immersive trend database.

2. Reproducibility: Immersive moodboards are datasets that can be experienced / duplicated on any basic immersive system.

3. Structural \& Parameter modification: Moodboards can be modified, reused and tweaked with as the digital support enables a quick modification of the overall ambiance (fog, color filters...) or of individual image (position, photo editing...)

The clearest effect observed is the emotional boost triggered by the interactive relationship between the designer and the trend data. As an early representation the immersive moodboard is an externalization of an ambiance. By externalizing the ambiance, the designer is able to experience the induced emotions. Thus the designer can understand and emotionally asses his work, involving the emotional processes of his brain in the aesthetic judgment.

Even before having given body to the product, the immersive moodboards tool validates guidelines and gives guaranties of design trails and design hypothesis. Augmenting the communication canal between the designer and his trend externalizations extends the fidelity of the designer's decisions through a better dialog with his representation.

To obtain valid evaluation of a trend, the system tricks the brain into thinking it is immerged in a reality. The immersive moodboards are intended so the designer is fooled into enacting with a virtual universe. When using a traditional moodboard, the designer is supposed to project himself in the ambiance he has created. Our system liberates the designer from this effort by projecting his consciousness in the moodboard through bodily engagement, giving a vivid feel to the trend represented. Exploring the immersive moodboards with one's body gives credibility to the early representation. The more credible the trend seems, the more truthful the judgments and style decision will be. The enactive link between consciousness, body, reality and ambiance serves the design process in trend evaluation.

It is interesting to see that modifying the support of the trend information only scarcely modifies how the designer attributes semantics and emotion terms to a trend. Our research has shown that the meaning communicated by a traditional moodboard and its immersive homologue are fairly similar. Furthermore we have measured that variance between the semantic profiles of immersive moodboards are $40 \%$ lower than that of the traditional moodboards. This indicates that spatialized virtual environment, as a support for trend information, prevents the semantic erosion inherent to the transition of a message from one support (moodboard) to another (designer). Using this tool will provide similar design context, similar mental representation anchors, and formal 
vocabulary basis and thus guaranties cohesion of the design team on the vision of the project.

With the right technological platform, an immersive moodboard is as simple to design as a traditional moodboard. The time investment is slightly increased since the third dimension adds to the complexity of the construction. However the raw material used to generate a traditional moodboard and an immersive moodboard are similar. The escalading development of three-dimensional ready hardware will soon enable this tool to enter design studios through lighter support. A few prototypes of 3D touch tablets are already in development and will surely suit the designers' needs.

The abstraction in early representation is significant because the early representations are an inspirational basis for the generational phase must question rather than answer the brief. The immersive moodboard is composed of images coming from very different sources (internet data bases, design journals, photographs...). Even though these images are close in style, their difference gives a surrealist feel to the overall composition. This fuzziness opens the possible solution space and engages the designer to filling the design gap.

\section{Conclusion}

The immersive moodboard application is one more application added to the digital design workflow developed by the New Product Design Laboratory. This experiment gets us one step closer to understanding how the final product kansei identification can be augmented by virtual reality techniques during early design phases. Through the exploration and the inspirational phases, the designer works on the kansei as an object helping to predict the future impact of the product. Spatializing the trend information and immerging the industrial designer very soon in an early representation of the final product has proven to be impactful and to augment bodily and emotional engagement. The immersive moodboard as a tool must be a design proposition used as a basis to question the impact of the future final product. In so far as a moodboard is shared within the design studio, having a media that gets the designers to consider the inspirational space with a holistic and coherent vision in which all composition pieces (individual pictures \& billboards) are interacted on a realistic spatial level, enables designers to share the same references as a project starting point. By combining the reality of the designer in inspirational phase and that of a set of trend data, the confrontation is inevitable and the designer is tricked into seeing the moodboard as a plausible reality. This confrontation emulates the kansei of the future product. The traditional way of having the designer going towards a non-responsive media is over. The interactivity gives us access to stimulating and engaging design environments. Acting as an intermediate representation, the immersive moodboard boosts some characteristics of the traditional moodboards, such as: The ability to impact, stimulate, thrill, activate curiosity, interest and inspire the designer. This induced state of mind seems to correspond with the design exploration and questioning inherent to early design process. Having a vivid representation of the kansei references to the product design project forces the designer to profoundly confront himself with the environment, ambiance and design set up he has to work with. We believe that the emotional activity of an industrial designer during the 
inspirational and generative act, impacts his production. Rather than centering the design process on the end user only, we have developed tools and methods taking in account the industrial designer himself: Shifting the paradigm from a user centered design to a user \& designer centered design. We believe that immersion is a lever towards an attractive, engaging and efficient design process. 
A. Edwards, S. A. (2009). A Comparative Study of Developing Physical and Digital Mood.

A.Hatchuel, B. (2009). C-K design theory: An advanced formulation, Research in Engineering Design.

Alvarez, J. (2007). Du Jeu vidéo au Serious Game: approches culturelle, pragmatique et formelle.

Aubert, B. (1997). Les technologies de l'information et de l'organisation.

B.Bachimont. (2004). Arts et sciences du numérique : ingénierie des connaissances et critique de la raison computationnelle.

Bach y Rita. (1960). TVSS.

Bae. (2009). Ilovesketch SIGGRAPH 09.

Bassereau, J. (2001). De l'Ingénierie centrée sur la technique à l'Ingénierie.

Benayoun, M. " E-moving stills / Still moving ".

Berthouse, N. (2006). Corpus-Based Models Of Multimodal Behaviors.

Bertin, J. (1999). Sémiologie Graphique: Les diagrammes, les réseaux, les cartes.

Bonnamy, L. (2008). Le design sensoriel comme moteur.

Bouchard, C. (2005). L'analyse des tendances conjointes.

Bourassa, B. S. (2000). Apprendre de son expérience .

Bouvier, P. (2009). La présence en réalité virtuelle, une approche centrée utilisateur.

Burkhardt. (2003). Immersion, Réalisme et Présence dans la conception et l'évaluation des environements virtuels.

Buxton, B. (2007). Sketching user experience.

C. Woelfel, J. K. (2010). Knowing, Reasoning and Visualizing in Industrial Design.

Caron, G. (2005). Les modes d'achat d'un produit et de son packaging.

Chatonsky, G. (2008). "Contre l'immersion".

Childs. (2004). Cited in "Product Experience" by Hendrik N. J. Schifferstein.

Christofol, H. (1995). Modélisation systémique du processus de conception de la coloration .

Claudia Eckert, M. S. (2000). Sources of inspiration: a language of design.

Csíkszentmihályi, M. (1975). "Théorie du flow".

Cuisinier, F. (2011). Doctoriales du Design.

Design, C. C. (2010). Suppléance perceptive.

Desmet. (2002). Designing Emotions. Thèse de Doctorat, Université de Technologie de Delft. Desmeules. (2000).

DOMOVISION. (2009). Mode d'emplois.

Eckert, C. (2004). Signs, symbols, and subjectivity: An alternative view of the visual.

Ekman, F. (1978.). Facial Action Coding System: A technique for the measurement of facial movement.

Erner, G. (2009). Sociologie des tendances.

F.Sebbah. (2008). Master course.

Front Design. (2006). Sketch Furniture.

Fuchs. (1996).

Göbel, F. D. (1994). Virtual prototyping - An approach Using VR - Techniques. 
Godet, M. (2004). Manuel de Prospective stratégique.

Guenand, A. (2008). Design d'anticipation et qualités sensorielles du produit.

Hall. (1996). The Hidden Dimension. New York: Doubleday \& Co.

Harada, A. (1998). On the definition of Kansei. Modeling the Evaluation Structure of Kansei.

Hendrik, S. J. (2010). The experimental assessment of sensory dominance.

Hollnagel. (2009). ETTO Concept.

Huong Q. Dinh, N. W. (2009). Evaluating the impotance od multi-sensory input on memory and the sense of presence in virtual environement.

Izard. (1977). Differential Emotions Scale.

Jakobson. (1960). «Closing statements : Linguistics and Poetics », Style in langage.

Jung von Matt, D. (2008). Tagged in motion.

Kadri, A. (2007). Contribution de la réalité virtuelle à l'évaluation de produits, dans les phases amonts du processus de conception.

Kim, J. (2011). Modeling cognitive and affective processes of designers in the early stages of design: mental categorization of information processing. Paris: Thèse Pastel.

Lebahar, J. (2007). La conception en design industriel et en architecture. Hermès - Lavoisier.

Legendre. (1993).

Lenay, C. (2008). Perception \& Enaction Cours UTC Master ICI.

Lévy, P. (2008). L'ingenierie kansei ou comment lier affectivite et conception.

l'ingénieur, D. s. (2009). Louise Bonnamy.

Lucero, M. (>2005). Mood Boards: Industrial Designers" Perception of Using Mixed Reality.

Ludden, G. S. (2011). Beyond surprise: a longitudinal study on the experience of visual - tactual incongruities in products.

M.Minsky. (1965). Matrices de Découvertes.

Marc, D. (2006). Approche sémiotique de l'interface matérielle et logicielle.

Mehrabian, R. a. (1977). Scale for pleasur and arousal.

Mel Slater, V. L. (1969). "Immersion, Presence, and Performance in Virtual Environments ".

Mougenot C., A. J. (2010). Comparing the effects of visual and auditory stimuli in design creativity.

Third International Workshop on Kansei.

Mougenot, C. (2008). Towards stimulating creativity in design: A model of informational activities in early phases of product design process.

Nagamachi. (1997). Kansei classification.

Nagamachi. (1995). Mazda Miata Kansei Engeneering.

Nagamachi, M. (2002). Kansei engineering as a powerful consumer-oriented technology for product development.

Nagamachi, S. E. (2005). Affective meaning: The Kansei Engineering approach.

Nagasawa. (2002). Kansei and Business. Kansei Engineering International - International Journal of Kansei Engineering, 3, pp. 2-12.

Nelson, J. (2010). Travaux de thèse.

Norman. (2002). The design of every day things. 
Norman, D. (1988). The psychology of Everyday Things.

O.Assouly. (2007). Internet et la synthèse collective des goûts.

Olivier Gapenne, P. G. (2005). Suppléance perceptive et interface : une.

Ortony, Norman, \& Revelle. (2004).

Osgood. (1957). Differential Sémantic Scale.

P. Fuchs, G. M. (2006). Traité de la réalité virtuelle.

P.Levy. (2008). Ingénierie Kansei, ou comment lier affectivité et conception .

Padilla. (2001).

Picard, R. (1997). Affective Computing. The MIT Press, Cambridge, MA.

Project, 3. (2011, 01). Designing team. (V.Rieuf, Intervieweur)

Renault. (2001). Talisman Concept Car.

Rieuf.V. (2007). Analyse des processus psychologiques de création: Etude de croquis. U.Nimes.

Rockeach, S. e. (1968-2010). Synthèse des listes de valeurs.

Rouach, D. (2005).

Rouveray. (2006). Iintergation des Preferences Emotionelles et Sensorielles dans la Cconception de Produite d'ameublement.

S.Ben-Mahmoud-Jouin. (1999). Stratégie d'offres innovantes et processus de conception.

Sasche. (2002). Idea materialis Entwurfsdenken und Darstellungshandeln: cited in Knowing reasoning and Visualizing in Industrial Design.

Schon, D. (1983). The reflective practitioner, Temple-Smith, London.

Schütte, S. (2008). Affective meaning: The Kansei Engineering approach.

Schütte, S. (2005). Engeneering Emotional Values in Product Design: Kansei Engineering in

Developpement.

Schütte, S. (2005). Engineering Emotional Values in Product Design: Kansei Engineering in

Development.

Schütte, S. (2005). Engineering Emotional Values in Product Design: Kansei Engineering in

Development.

Sebat, F. (2009). Cours UTC Master ICI.

Shannon, W. (1978). Théorie de l'information et de la communication.

Song, E. (2010). Travaux de thèse.

Thouvenin, D. L. (2008). Cours UTC MAster ICI.

Troussier, X. F. (2004). La Réalité Virtuelle pour une conception centrée sur l'utilisateur.

Uhlmann, W. \&. (2008). Two-Step-Method.

Weber, B. (2010). L'arbre des possibles .

Witmer, B. \&. (1998). Measuring presence in virtual environments : A presence questionnaire.

Won, P. (2001). The comparaison between visual thinking using computer and conventional media in the concept generation stages of design.

Y. Shibata, A. (2003). Kansei Information Processing and Virtual Reality Techniques for Japanese Traditional Crafting Presentation". 